\title{
DEL CONFLICTO AL AGONISMO. LA OPERACIÓN CLAVE DE LA DEMOCRACIA $^{1}$
}

\author{
Pauline Colonna d'Istria ${ }^{2}$
}

\section{Resumen/abstract}

Una aproximación irreductiblemente conflictiva a la democracia parece conducir necesariamente a una relación esencialmente negativa de la política. Como si existiera una suerte de antinomia entre una comprensión radicalmente conflictual de lo político y la concepción de un nuevo proyecto democrático. Si algunos teóricos agonísticos confirman y justifican aún esta imposibilidad, los análisis de Chantal Mouffe abren nuevas perspectivas. ¿De qué manera Chantal Mouffe logra articular una comprensión maximalista del conflicto y la elaboración de un nuevo proyecto político?

Palabras clave: democracia agonística, conflicto político, antagonismo, neoliberalismo, democratización

FROM CONFLICT TO AGONISM. THE KEY OPERATION OF DEMOCRACY

A radically conflictual approach of democracy seems necessarily to nurture a negative posture towards politics. As if there were a kind of antinomy between an agonistic understanding of politics and the elaboration of a new democratic project. If certain agonistic theorists confirm and even justify such an impossibility, Chantal Mouffe opens new perspectives. How does she succeed in combining a maximalist interpretation of conflict with the elaboration of a new political project?

Keywords: agonistic democracy, political conflict, antagonism, neoliberalism, democratization

\footnotetext{
${ }^{1}$ Traducción del francés de Cristina Hurtado.

2 Francesa. Universidad de Paris Nanterre La Défense. E-mail: p.colonnadistria@hotmail.com 


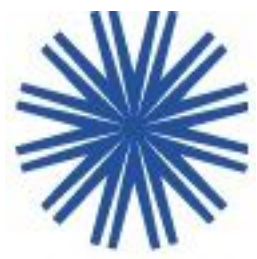

A menudo el aporte de los trabajos de Chantal Mouffe a la teoría política contemporánea es identificado como contribución a un entendimiento irreductiblemente conflictual de la política democrática. Chantal Mouffe ha llegado a ser, de hecho, una de las principales representantes de lo que se llama la "democracia agonística". Este marco teórico alternativo a aquel que domina ampliamente hoy día la teoría política -el de la democracia deliberativa- no renvía, sin embargo, a una escuela de pensamiento unívoca, identificable a un proyecto teórico político bien definido. Los teóricos llamados "agonísticos" declaran fuentes diversas y defienden concepciones diferentes, a veces hasta opuestas, del espacio público, del debate democrático o de la acción política en su relación con las instituciones. Por lo que podemos considerar que el concepto de "democracia agonística" se refiere más bien a una prioridad que a una fuente, siendo esa prioridad el conflicto en los análisis de lo político y a una política democrática en particular.

Por lo tanto, si existen al menos tantas concepciones diferentes del agonismo democrático como las hay, por otro lado, de las variantes de democracia deliberativa, es debido también a que este "conflicto", al cual se quiere darle un lugar central y fecundo, no es en absoluto unívoco. Todos los pensadores que han contribuido de manera decisiva a replantear la cuestión democrática partiendo de la noción de conflictualidad no privilegian de la misma manera los términos ni las ideas. Ed Wingenbach, que propone la tipología más convincente de la cual podemos disponer para orientarnos en esta literatura agonística (Wingenbach 2011) identifica la presencia de dos grandes tendencias, es decir, dos "campos" distintos organizados en torno a la noción de "resistencia", por un lado, y de "pluralismo" por otro. Las teorías agonísticas de la democracia se distribuyen entre los "agonismos de resistencia" y los "agonismos pluralistas", a los cuales se puede agregar, por otro lado, un "agonismo oposicional" y un "agonismo expresivo", defendido sobre todo por Bonnie Honig y David Owen (Honig 1993; Owen 2009) quienes insisten sobre las virtudes de la oposición y de la competencia en sí misma. Y, de otro lado, un "agonismo constitucional” como lo teoriza James Tully (Tully 2008), y un "agonismo receptivo" (responsive agonism) desarrollado 
el "agonismo del adversario" representado por Chantal Mouffe que propone otro análisis de las dinámicas institucionales de lo político. Los políticos democráticos señalados divergen según insisten en la "oposición" o en la noción de una división irreductible o sobre la pluralidad. Si seguimos a Ed Wingenbach, los agonismos que ponen el acento en el pluralismo serían los únicos que proponen modelos "productivos", que inspiran la investigación en ciencias políticas, mientras que los agonismos centrados sobre la importancia de la resistencia o de una contestación permanente, o que otorgan al conflicto una definición "maximalista", de cierta manera no llegarían a superar la formulación de certezas negativas ni a alimentar eficazmente la concepción de nuevas políticas democráticas. Ahora bien, lo que olvida la tipología de Wingenbach es el agonismo teorizado por Chantal Mouffe que nos parece que ocupa un lugar diferente.

Chantal Mouffe, en efecto, propone una definición del conflicto político mucho más radical que aquella que encontramos en el horizonte de los trabajos de Tully o de Connolly, a los cuales se encuentra asociada en este caso. La idea de un pluralismo sin antagonismos es para ella una quimera. Y si el modelo de democracia que ella elabora nos parece especialmente interesante, es justamente porque da al conflicto una "carga máxima" para retomar la imagen del filósofo Miguel Abensour (1993) y se construye sobre el análisis de una dimensión verdaderamente ontológica del conflicto, pero sin renunciar a explicitar ciertas distinciones que nos permiten precisar el contenido de una política conflictiva de emancipación. Esta doble postura representa casi un desafío en el seno de la literatura agonística, tanto que parecería existir una verdadera antinomia entre una comprensión irreductiblemente conflictual de lo político y la concepción viable de un nuevo proyecto democrático que ponga en juego transformaciones efectivas. Si seguimos autores como Slavoj Żižek o Alain Badiou, y no sólo a ellos, una aproximación agonística de lo político sería incapaz de superar el statu quo reinante y terminaría aceptando la democracia liberal tal como es. La deconstrucción a la cual daría pie este pensamiento irreductiblemente conflictual de lo político funcionaría, en realidad, "como puro y simple veredicto de una imposibilidad militante" (Badiou 1998). Los análisis de Mouffe hacen, pensamos, la demostración inversa. ¿De qué manera la aproximación conflictual de Mouffe escapa a la antinomia y logra articular una comprensión maximalista del conflicto en la elaboración de un nuevo proyecto político? 
El lugar que Chantal Mouffe otorga al conflicto político no es ni provisorio, ni de cierta manera, estrictamente antropológico. La centralidad que le confiere va más allá de un reconocimiento de hecho a la pluralidad inevitable de opiniones y de modos de vida -lo que con Rawls podemos llamar "los hechos del pluralismo"- y a su carácter irreductible. No se refiere solamente a la "tendencia a la agresión presente en todos los seres humanos" (Mouffe 2005: cap. 2) que el psicoanálisis nos fuerza a reconocer y que según éste la teoría democrática no consideraría suficientemente. Chantal Mouffe afirma la existencia de un conflicto mayor, estructurante, y deduce, por decirlo de alguna manera, que por sobre los antagonismos particulares, hay una verdadera lógica del conflicto que es la condición misma de una sociedad política.

Mouffe, lo sabemos, parte de una definición schmittiana de la política, de una definición de la política por el antagonismo, haciendo del trazo que separa amigo/enemigos el gesto característico por el cual se define una sociedad política. La democracia no podría pretender superar esta división primera, pero le es propio establecerla de manera diferente. Es necesario darse cuenta que Mouffe no retiene de Schmitt los términos de la relación que describe, sino la relación misma: es la naturaleza relacional de las identidades políticas lo que le interesa, el hecho que la creación de un "nosotros" depende siempre de la distinción con "ellos". Es por eso que partiendo de la intuición schmittiana le da una formulación derridiana al insistir sobre la diferencia y no sobre la enemistad. En efecto, el término "exterior constitutivo" expresado por Henry Staten en referencia a ciertos conceptos de Derrida (la "huella", el "suplemento", la “diferencia”) le parece, finalmente, más pertinente, ya que considera que toda identidad es relacional y que la condición de existencia de una identidad es la afirmación de una diferencia, es decir, la determinación de un "otro que no es ella" y que funciona como su “exterior" (Ibíd.) El antagonismo no está simplemente en la sociedad: es generador de su constitución; dicho de otra manera describe el modo de institución de la sociedad, su condición misma de posibilidad. Definiendo la política por "el momento del antagonismo", Mouffe nos pone en presencia de una especie de argumento trascendental que hace aparecer el discurso de la conflictualidad en un sentido muy diferente de donde lo sitúa, en general, la teoría política cuando analiza los diferentes conflictos en las 
sociedades pluralistas, y que busca limitar los vectores de desacuerdo. Si los conflictos, insiste Mouffe, no están en miras de desaparecer, es porque el antagonismo es la condición misma de la política y de la sociedad. Es, en esta perspectiva, postfundacionalista que analiza correctamente Oliver Marchart (2007), el fundamento negativo de la comunidad política. En cierto sentido el antagonismo no reenvía ni a oposiciones reales ni a contradicciones lógicas. Como lo explicaba ya con Ernesto Laclau en Hegemonía y estrategia socialista: "el antagonismo, lejos de ser una relación objetiva, es una relación en la cual se muestran los límites de toda objetividad" (Laclau y Mouffe 2001: 230); el antagonismo es "la experiencia del límite de lo social”(Ibíd.: 231), este límite, en vez de ser provisorio, se transforma en constituyente. La definición que Mouffe da del antagonismo nos pone en presencia de una verdadera ontología política que la aproxima sobre todo al pensamiento conflictual de la democracia desarrollada por Claude Lefort, a partir de una reflexión sobre la "división originaria de lo social" y no de teorías agonísticas de la democracia como las desarrolladas por Bonnie Honig o de James Tully. Esta comparación es interesante, independiente de que Mouffe se refiera a menudo a Claude Lefort, porque muestra las dificultades a las que puede conducir una concepción de lo político que hace del conflicto la condición estructural de toda sociedad.

En cuanto la concepción lefortiana de la democracia, como democracia necesariamente conflictual, lo que retiene la atención en el seno del campo actual del pensamiento político, es que Lefort describe un régimen o una forma de sociedad en la cual los conflictos son muchos más profundos que aquellos que confronta el pluralismo de las sociedades modernas. Mouffe insiste sobre un conflicto irreductible, instituyente, que escapa a las referencias empíricas de los conflictos de hecho. A partir de una relectura apasionante de Maquiavelo, Lefort (1972) afirma la existencia de un conflicto mayor cuyo núcleo aparecería como "disimulado" pero del cual dependen el mantenimiento y la invención de las democracias. La democracia se instituye y se reinventa a partir de sus divisiones que es necesario referirlas, para comprenderlas, a una división "originaria" siempre actuante del espacio social. No podemos entrar en el detalle de sus análisis, pero el hecho es que ha podido suscitar una forma de desconfianza por "el énfasis ontológico" que operarían en el lugar de la conflictualidad, para retomar una crítica formulada por Jean-Marc Ferry. Lefort haría "trascendente" un "rasgo notable de las sociedades modernas"; sería, escribe, "como un énfasis ontológico realizado sobre el hecho 
sociológico que es el pluralismo de nuestras democracias occidentales contemporáneas" (Marchart 2007: 159).

A lo que se refiere es a un "énfasis" que relativizaría la fuerza crítica de su conceptualización del hecho democrático y, por efecto de generalización, a la significación de la reflexión sobre la conflictualidad social. Como si la insistencia sobre la irreductibilidad del conflicto empujara a considerar todo proyecto de consenso como mortífero - una definición "máxima" del conflicto podría conducir a valorizar la conflictualidad "en sí misma", sin permitirnos distinguir claramente los conflictos creadores de dinámicas destructivas de lo político. Como si al plantear el conflicto en tanto condición propia de las sociedades políticas, nos correspondiera el "conservar" este desfasaje, vigilar para que la diferencia no sea absorbida y preservar así la posibilidad de un dinamismo histórico. A pesar de todos los matices que caracterizan el pensamiento de Claude Lefort, es impresionante ver que la insistencia sobre el carácter mortífero de los que niegan el conflicto domina la reflexión sobre las modalidades de institucionalización democrática del conflicto. Lefort retiene de la lectura de Maquiavelo que "la mejor república (...) no da una solución al problema político. Se distingue más bien por un abandono tácito de la idea de solución, a causa de la recepción que ella hace a la división y, bajo el efecto de ésta, al cambio y, a la vez, a las oportunidades que ofrece a la acción" (Lefort 1992: 175). Por lo tanto ¿basta con "liberar” el conflicto, haciéndolo visible a los ojos de todos (es decir recordando la contingencia radical de todo orden social) para reabrir automáticamente nuevas vías a la democracia? ¿Basta levantar el velo de la negación de la conflictualidad y de "acogerlo"? La elección de interrogar el conflicto en su dimensión ontológica ¿no conduce a abandonar una reflexión política concreta sobre las modalidades de recepción y de potencialidades mortíferas, pero igualmente creadoras?

Adoptar un punto de vista agonístico no conduce necesariamente, como lo pretende Żiẑek, a la justificación de un status quo, pero, es verdad, que aparece en numerosos pensadores del conflicto democrático como un "apriorismo emancipatorio" (Marchart 2007: 159), poniendo en juego una confianza en la lógica subversiva propia de la lógica democrática. Por poco que se los siga, cuando se encuentran frenados los principios de libertad e igualdad de los cuales la democracia es portadora, los conflictos 
servirán siempre de motor de emancipación. Si bien Chantal Mouffe considera igualmente que la creación de un espacio agonístico de resistencia es la "condición sine qua non de una práctica efectiva de la democracia” (Mouffe 2005: introducción), ella explica que la transformación de antagonismos sociales en conflicto políticos creadores supone una operación -y la idea misma de agonismo es el resultado de esta operación. Consciente del "carácter polisémico de todo antagonismo" (Mouffe 2005: 20), nos da preciosos instrumentos para redefinir el contenido de una política democrática de emancipación.

Encontramos en Mouffe escasamente el término "conflicto" como se usa en general. La noción de "agonismo" que prefiere, no lo sustituye simplemente; es el resultado de una redefinición de lo que se podría entender por "conflicto", lo que le permite precisar el género de una conflictualidad que una política democrática debe promover. Mouffe nos invita a distinguir dos modalidades de relación conflictual: uno que opone enemigos, la otra confronta adversarios; el primero describe un antagonismo, el segundo un agonismo. El conflicto es el criterio apropiado de lo político, su "diferencia específica" para decirlo en los términos de Schmitt, pero esta diferencia específica no toma necesariamente la forma de antagonismo. Pertenece, precisamente, a la democracia evitar que el conflicto no se transforme en esta forma particular de oposición. La democracia, explica Mouffe, debe transformar el antagonismo en agonismo.

\footnotetext{
"Para que pueda ser considerado legítimo, el conflicto necesita tomar una forma que no destruya la asociación política. Esto significa que debe existir entre las partes en conflicto un cierto tipo de lazo común, de manera que no visualicen a los opositores como enemigos a erradicar, percibiendo sus demandas como ilegítimas -lo que sucede en la relación antagonista amigo/enemigo. Los opositores no pueden sin embargo ser considerados como simples competidores cuyos intereses podrían ser tratados por medio de una negociación o reconciliación a través de una deliberación. En ese caso, el elemento antagonista seria simplemente eliminado. Si se quiere reconocer la permanencia de la dimensión antagónica del conflicto, por un lado, y a la vez hacer posible una "domesticación" por otro, sería necesario concebir un tercer tipo de relación. Es el caso de la que he propuesto llamar "agonismo." (Mouffe 2005: 20).
}

El agonismo, tal como Mouffe lo describe aquí, aparecería como una forma de "amansamiento" del antagonismo. No es ni su contrario ni tampoco su prolongación natural: es el resultado de un proceso de transformación, de doma, que le confiere a la vez 
su legitimidad -legitimidad que no se establece a partir de criterios exteriores, sino que depende del hecho que el conflicto no pone en peligro la asociación política. El argumento de Mouffe nos muestra que no es contradictorio insistir, a la vez, en las dinámicas conflictuales de lo político y en su dimensión de asociación. Al contrario, es no perdiendo jamás de vista las condiciones de posibilidad, aunque sean mínimas de una asociación política, que los conflictos devenir "motores" de crecimiento de la democracia, como Lefort pudo escribir en otro ensayo. Mouffe nos muestra que es posible construir una reflexión sobre el análisis de la dimensión ontológica del conflicto sin dejar de lado el nivel óntico donde se ejerce la política. Con el ejemplo de Lefort, que escogió separar el concepto de política oponiendo "la" política (como campo empírico, objeto de la ciencia política) y "lo" político (en su esencia como modo de institución de la sociedad), Mouffe distingue dos niveles de la política -el nivel óntico y el nivel ontológico, redefiniendo dos usos del término- "politics" de un lado y "the political" del otro, pero no abandona jamás un término en beneficio del otro. A pesar de que considera que "es la falta de comprensión de qué es lo político (the political) en su dimensión ontológica lo que está al origen de nuestra incapacidad actual de pensar de una manera política", ella precisa que "su terreno de investigación" se sitúa prioritariamente en el nivel "óntico": su interés mayor es la política (Mouffe 2005: 9). Su reflexión sobre la esencia de lo político está al servicio de una interrogación sobre el devenir de la democracia, cuya fuerza crítica se encuentra ligada a una propuesta. Las distinciones realizadas por Mouffe entre el antagonismo y el agonismo, entre las figuras del enemigo, competidor y adversario, son instrumentos preciosos para pensar una política democrática conflictual. Las diferencias que establece entre conflicto y violencia, o a la inversa, el esfuerzo para permanecer unidos disensión y asociación nos permite precisar la forma del conflicto a la cual se enfrenta la democracia y qué transformación debe realizar una política democrática para que el conflicto sea benéfico. Una comprensión conflictual de la democracia debe poder conducir a una nueva política transformadora. Para esto, Mouffe no se contenta en pensar la institucionalidad simbólica del conflicto en democracia, sino que reflexiona concretamente en los procesos institucionales que pueden hacer el conflicto operante. El paso del antagonismo al agonismo que describe como sublimación es la obra de las instituciones. En efecto, la institucionalización es el principal medio por medio del cual se encuentra desarticulado el potencial siempre presente del antagonismo inherente de las relaciones sociales: "los conflictos antagonistas son menos susceptibles de emerger cuando existen circuitos políticos legítimos para las voces disidentes. Si no los desacuerdos tienden a tomar formas 
violentas, y esto vale tanto en política interior como en política internacional" (Mouffe 2005: 20-21).

La lectura que Mouffe hace de las instituciones y dispositivos que regulan su funcionamiento es muy original: en vez de insistir en la regulación que suministran las instituciones y el dominio de las pasiones que permiten, subraya la dimensión finalmente catártica de la institucionalización. Si la transformación del antagonismo en agonismo que describe como el principal objetivo de la política democrática, depende mucho del vigor de las instituciones parlamentarias, su buen funcionamiento está ligado a la polarización de las pasiones que se desarrollan en su seno, alrededor de oposiciones aparentes. Dicho de otra manera, las instituciones deben permanecer en un terreno de enfrentamiento y por lo tanto representar las fuerzas y los proyectos contrarios. Lo que Mouffe valoriza en el sistema parlamentario, es menos su forma de proceder como su carácter partidario: la presencia de identidades políticas competitivas es fundamental, porque ello permite encontrar una especie de salida democrática a la expresión de las pasiones. Podemos estar sorprendidos del carácter estructurante que Mouffe reconoce a la oposición derecha/izquierda en el campo democrático porque estamos acostumbrados a entender que la polarización bipartita de la vida política constituye un cerrojo arcaico a la emergencia de una verdadera política democrática. Pero Mouffe no promete el retorno a la política de partidos tradicionales: insiste en la reconstrucción necesaria de verdaderas alternativas que encarnen proyectos políticos diferentes. Como sea, para comenzar a redefinir los contornos de una política democrática agonista, no es necesario invertir únicamente en los espacios extra-parlamentarios o buscar la solución en una "subpolítica" liberada de los partidos y de identidades de clase, sino de permitir a las voces disidentes poder expresarse, entrar políticamente en competencia en el espacio parlamentario para que dicha política reencuentre su sentido y las potencialidades cuya escena supone. Cuando nosotros comparamos sobretodo su aproximación a Claude Lefort, que hace de la escena del conflicto la esencia misma de lo político, hemos explicado que la fuerza crítica de los trabajos de Mouffe proviene que en ella se encuentra ligada a propuestas. La idea de una democracia agonista representa una alternativa teórica a los modelos liberales, agregativos y deliberativos de la democracia que nos ofrece hoy la teoría democrática, pero ella articula un verdadero proyecto político, de una democracia radical y plural que representa una "alternativa para una nueva izquierda". Mouffe 
rechaza limitar las prácticas agonistas de lo político a una "estrategia de oposición” y las razones que da son especialmente instructivas.

Una comprensión agonista de la democracia no excluye la posibilidad de transformación política y socio-económica efectiva. Allí, el pensamiento de Mouffe permite salir de la antinomia que pretende la imposibilidad de instituir en un nuevo orden social si no es partiendo de cero, como única posibilidad de una "estrategia de oposición". La manera en que Mouffe ha presentado con Laclau las modalidades del proyecto de una democracia radical permite hacer surgir un elemento crucial: no se trata de escoger entre una "estrategia de oposición" y una "estrategia de construcción" sino de llegar a articular las dos. Cada estrategia, considerada en sí misma, es incompleta y arriesga petrificarse en un solo sentido, lo que comprometería la profundización de la dinámica democrática. En el caso de una "estrategia de oposición" domina el elemento de negación, "pero este elemento de negatividad no se acompaña de ninguna tentativa real de establecer puntos nodales diferentes desde los cuales podría surgir un proceso de reconstrucción del tejido social, diferente y positivo -y en consecuencia está condenada a la marginalidad": en el caso de la estrategia de construcción de un orden nuevo, al contrario, "el elemento de positividad social predomina, pero esta predominancia produce un equilibrio inestable y una tensión constante con la lógica subversiva de la democracia" (Laclau y Mouffe 2001: $323)$.

El término central a partir del cual se aclara el proyecto político de Mouffe es el de hegemonía. Es, por decirlo de algún modo, el término sin el cual la reflexión sobre el antagonismo en política no podría encontrar una salida práctica y permitir así una nueva política democrática. Y constituye para Mouffe el punto ciego de las formas antagónicas teorizadas por Honig y Connolly. La política de la resistencia que ellos promueven deja en la sombra un elemento determinante de las luchas: "el establecimiento de una cadena de equivalencias entre las demandas democráticas y la construcción de una hegemonía alternativa". "Desestabilizar los procesos dominantes y perturbar los acuerdos existentes no basta para radicalizar la democracia” (Mouffe 2013: 14) concluye; es necesario juntar dos dimensiones centrales de la política: el antagonismo y la hegemonía. 
No vamos a retomar la genealogía del concepto de hegemonía tal como lo han reconstruido Laclau y Mouffe. Pero está claro que este concepto permite a la vez una mejor visión de la dinámica democrática y un principio de orientación de la acción política: "Revela que es siempre posible cambiar las cosas políticamente y de interferir en las relaciones de poder para transformarlas" (Mouffe 2000: 119).

La democracia no está amenazada por la confrontación infinita de prácticas hegemónicas diferentes, sino al contrario por la ausencia de proyectos contrahegemónicos. Las instituciones democráticas están en peligro cuando se cede a la idea de que no hay alternativas y que no hay en el espacio político proyectos hegemónicos competitivos. Esto es válido tanto a nivel nacional como a nivel global, en tanto el "dogma de no hay alternativa" se justifica ahora en nombre de la globalización. Porque la mundialización no ha conducido a un pluralismo de hegemonías sino a reforzar un mundo unipolar, ha pasado a ser un factor extremadamente poderoso de des-democratización de nuestras sociedades. La reflexión de Mouffe sobre el agonismo político no se limita a escala nacional. Tomando el cuidado de precisar que ella no pretende "imponer" su modelo agonístico en el campo de las relaciones internacionales, subraya sin embargo su interés en una aproximación que tenga en cuenta las similitudes que presentan ambos espacios. Es en los dos niveles, nacional e internacional, donde la radicalización de los antagonismos nos muestra las condiciones para repensar las modalidades efectivas de una política democrática que desarme la violencia de los conflictos. Si seguimos la argumentación de Mouffe, se comprende que la transformación de antagonismos en agonismos democráticos depende de una pluralización de hegemonías. Un mundo unipolar es el terror de los conflictos nihilistas.

Mouffe argumenta que el fin de la guerra fría y la imposición de un modelo neoliberal de globalización coincide con la multiplicación de ataques terroristas de una manera que no es fortuita. ¿El terrorismo sería consecuencia de un mundo unipolar? Inspirándose en los análisis schmittianos relativos al estatus de las políticas pos-estatales, Mouffe propone una aproximación al terrorismo contemporáneo que rompe 
profundamente con la representación de un radicalismo fanático de grupos aislados: "se puede considerar el terrorismo como el producto de una nueva configuración de lo político, característico del tipo de orden mundial que se instaura alrededor de la hegemonía de una sola super-potencia” (Mouffe 2005: 81).

Dicho de otra manera, es indispensable elaborar proyecto y prácticas contrahegemónicos que alimenten un debate democrático, sin el cual el potencial antagónico de las relaciones sociales arriesga de cristalizarse en un violento rechazo no negociable. La expansión de la racionalidad neo-liberal nos impone la necesidad de repensar el lugar de los conflictos en nuestras sociedades, de hacer consciente las consecuencias políticas que dicha expansión induce. Como explica bien Étienne Balibar, la racionalidad neo-liberal representa una "mutación de la naturaleza misma de la actividad política": "no solamente en el sentido que ella tiende a neutralizar tanto cuanto sea posible el elemento conflictual (...) sino que quiere privar de antemano todo significado, y crear las condiciones de una sociedad en la cual las acciones de los individuos y de los grupos (comprendidas aquellas que son violentas) no obedezcan más que a un solo criterio: la utilidad económica. No se trata tanto, de hecho, de política sino de anti-política, de neutralización o de abolición preventiva del antagonismo sociopolítico" (Balibar 2010: 38). Su análisis, además, nos llama la atención sobre la "crisis de representación" que afecta los sistemas políticos contemporáneos. Balibar afirma que la crisis de representación como tal, la “descalificación del principio mismo de representación" es "el otro aspecto de transformación de lo político que se puede asignar al neo-liberalismo" (Ibíd.: 47).

Por un lado, se supone que aquella ha devenido inútil, "irracional, a causa de la emergencia de formas de "gobernanza" que permiten calcular y optimizar los programas sociales y los procesos de reducción de los conflictos sociales, en función de su utilidad, y por otro lado, se proclama, más que nunca, que la representación es una forma política impracticable, peligrosa, cuando la responsabilidad del "ciudadano-sujeto" se define antes que nada en términos de normalidad y de desvío, en relación a la norma social que se trata de controlar, y no de expresar o de dejar que se exprese dándole una "voz" (lo que quiere decir que "el odio a la representación" es también una forma de la "rabia a la democracia") (Ibíd.: 48-49).

El neoliberalismo desactiva no solo la base misma de la democracia dejando casi caduca la capacidad de una delegación de poder y de control de los resultados de la delegación sino también la idea misma de "poder soberano". Balibar insiste y nos advierte sobre la importancia de "combinar" de manera inédita la espontaneidad y la institución, la participación y la representación" (Ibíd.: 49) para luchar contra la disolución de las 
estructuras de la ciudadanía y para inventarle nuevas modalidades. Pero esta "nueva política insurreccional" (Ibíd.: 49) no es por ahora sino imaginada. Balibar bosqueja nuevas "esperanzas" como lo escribe el mismo, pero no tanto en relación a nuevas alternativas políticas que nos permitirían concebir qué "nuevas políticas insurreccionales" son las más adecuadas para oponerse a las lógicas problemáticas que debilitan la democracia. En Chantal Mouffe podemos encontrar más explicitadas las modalidades concretas de una política conflictual en un contexto de hegemonía neoliberal. Profundizando las pistas de reflexión que ella nos ofrece podremos comprender los desafíos mayores de una concepción agonista y no sólo poner a prueba el neoliberalismo, sino como una de las respuestas posibles al servicio de un proyecto de democracia radical y plural. El agonismo que defiende está desde el comienzo puesto como una solución a la ofensiva antidemocrática de la "nueva derecha" neo-liberal, en todo el mundo y es lo que confiere a sus análisis tanta importancia. Cierto, Mouffe reconoce que la situación actual no es la misma que cuando ella escribió con Laclau Hegemonía y estrategia socialista. "En cuanto a la posibilidad de radicalizar la democracia, escribe ella, la situación hoy día es desgraciadamente menos favorable que como lo era hace treinta años cuando escribimos el libro". En una entrevista con Elke Wagner, dice "En vez de luchar por una radicalización de la democracia, debemos reducirnos a pelear contra el desmantelamiento de las instituciones democráticas fundamentales" (Mouffe 2008). Pero las recomendaciones realizadas más arriba, permanecen válidas: es necesario crear cadenas de equivalencias entre las diferentes luchas progresistas y crear las mediaciones institucionales indispensables para poner en cuestión el orden hegemónico actual.

En este texto nos concentramos en las modalidades de esta "alternativa para una nueva izquierda" que Mouffe explicita sobre la posibilidad de articulación entre una comprensión irreductiblemente conflictual de lo político y la elaboración de un nuevo proyecto democrático. Porque esta articulación misma no es nada evidente, nos parece decisivo recordar la idea de "democracia agonista" que puede suscitar, tanto de un lado como de otro, resistencias.

En este debate de gran importancia al interior de la teoría política es donde se confrontan concepciones deliberativas y concepciones agonistas de la democracia y a 
menudo se imponen oposiciones estériles: como si la reflexión sobre el pluralismo de las sociedades liberales pusiera frente a frente a los partidarios del consenso y a los partidarios del conflicto como si se tratara de un duelo en el cual hubiese una sola alternativa: "o bien el intercambio entre asociados discutiendo de sus intereses o normas, o bien la violencia irracional" (Rancière 1995: 72). Esta "falsa alternativa" que describe bien Jacques Rancière, termina por encerrar el debate de manera tal que los defensores de uno u de otro lado no lleguen jamás a darse cuenta de la trampa. El elogio enfático del "conflicto" indefinido sobre el cual reposa una gran parte de la literatura agonística impide en efecto construir puentes con las teorías competitivas y sobretodo de visualizar como sería posible profundizar la democracia, "radicalizarla" a partir de la comprensión irreductiblemente conflictual de sus dinámicas. La manera en que Mouffe define el agonismo político abre, según nosotros, otras vías.

Escoger interrogar el conflicto en su dimensión estructural, ligándolo al modo de institucionalización de lo social, no excluye reflexionar sobre sus diversas manifestaciones y sobre las diversas maneras de significar el conflicto. La definición de la democracia como una lógica subversiva no basta para afirmar que todo conflicto es un motor de emancipación y a confiar, en cierta medida, en las lógicas desarticuladoras de lo político: la reluctancia a comprometerse en el terreno institucional no se deriva de ninguna manera de la adopción de un punto de vista agonista de la democracia. Pensamos, al contrario que tal compromiso es necesario para las transformaciones mismas que el capitalismo impone hoy a las instituciones democráticas liberales. El giro neoliberal del capitalismo representa una fuerza de "des-democratización" que no se puede dejar de lado y que requiere no sólo de respuestas económicas y sociales sino también de nuevas respuestas políticas. En un contexto marcado por una desafección creciente frente a los político y una crisis de participación y sus efectos, conduce a la oposición -a falta de encontrar canales de expresión adecuados- a expresarse en los extremos del marco político, una concepción conflictual de la democracia es susceptible de encontrar una nueva intensidad. Los trabajos de Chantal Mouffe ofrecen una demostración convincente. La afirmación de la irreductibilidad esencial del conflicto no coincide necesariamente con la imposición de un impedimento a toda política militante o a todo proyecto político substancial. 


\section{Referencias bibliográficas}

Abensour Miguel (1993), "Democratice salvaje et enjarcies", Breve Euro Penes des Ciencias Sociales, Tome XXXI, No 97.

Badiou Alain (1998), Abrego de métapolitique, Seuil, Paris.

Balibar Etienne (2010), La proposición de l'egaliberté, PUF, Paris.

Connolly William (1991), Identity/Difference. Democratic Negotiations of Political Paradox, University of Minnesota Press, Minneapolis.

Press, Menneapolis and Londres.

(1995), The Ethos of Pluralization, University of Minnesota

Honig Bonnie (1993), Political Theory and Displacement of Politics, Cornell University Press, Ithaca.

Laclau Ernesto et Chantal Mouffe (2009) Hégémonie et Strategie socialiste. Vers une politique démocratique radical, Éditions Les Solitaires Intempesifs, Besançon.

Lefort Claude (1972) Le travail à l'óeuvre, Machiavel, Gallimard, Paris. (1992), Ecrire. À l'épreuve du politique, Calmann-Lèvy, Paris.

Marchart Oliver (2007), Post-foundational Political Thought: Political Difference

in Nancy, Lefort, Badiou and Laclau, Edinburgh University Press, Edinburgh.

Mouffe Chantal (2000), The democratic Paradox, Verso, London and New York. (2005), On the political, Routledge, London and New York. (2008), "Anagonisme et hégémonie. La démocratie radicale contre le consensus néolibéral”, La Revue Internationale des Libres et des Idées, $\mathrm{N}^{0} 3$.

\section{London and New York}

(2013), Agonistics. Thinking the World Politically, Verso

Owen David (2009), "The expressive Agon: On Political Agency in a Democratic Constitutional Polity, in Schaap (ed.) Law and Agonistics Politics, Ashgate, Farnham.

Rancière Jacques (1995), La mèsentente, Galilée, Paris.

Tully James (2008) Public Philosophy in a New Key, Volume I: Democracy and civic Freedom, Cambridge University Press, Cambridge.

White Stephen (2009), The Ethos of Law Modern Citizen, Harvard University Press, Cambridge.

Wingenbach Ed (2001), Institutionalizing Agonistics Democracy. PostFoundationalism and Political Liberalism, Ashgate, Farnham. 